\title{
FORMATIONS VÉGÉTALES ARBORÉES RIVERAINES DES COURS D'EAU ET POTENTIALITÉS PISCICOLES
}

\author{
H. PIÉGAY^ et Laurence MARIDET**
}

* Agence de l'Eau Rhône-Méditerranée-Corse (31 rue Jules Guesde, 69510 Pierre-Bénite) et Centre de Recherche en Géographie et Aménagement, Université Jean Moulin Lyon III (URA CNRS 260, 74 rue Pasteur, 69239 Lyon Cedex 02), France.

* CEMAGREF, Division Biologie des Ecosystèmes Aquatiques, Laboratoire Hydroécologie Quantitative (3 bis Quai Chauveau, 69336 Lyon Cedex 09) et Institut d'Analyse des Systèmes Biologiques et Socio-Economiques, Université Claude Bernard Lyon I, Laboratoire d'écologie des eaux douces et des grands fleuves (URA CNRS 1451, 43 Bd du 11 novembre 1918, 69622 Villeurbanne Cedex), France.

\section{RÉSUMÉ}

Les formations végétales arborées riveraines sont en étroite interaction avec le milieu aquatique environnant. Les conditions hydrodynamiques et morphodynamiques régissent la répartition des communautés végétales et réciproquement la végétation riveraine, par sa présence effective ou par ses apports en débris ligneux grossiers, est elle-même un élément structurel de l'hydrosystème. Ainsi, sa présence complexifie l'habitat aquatique, créant un milieu diversifié propice au développement des communautés biologiques et notamment piscicoles. Elle agit également directement sur la chaîne trophique en injectant dans le système des débris organiques, et indirectement en limitant la production autotrophe par ombrage, en régulant les apports en nutriments et la température. En raison du fort degré d'interconnexion entre le milieu aquatique et le milieu terrestre environnant, il est important d'inclure dans les préoccupations de gestion des hydrosystèmes celle des corridors végétaux sur l'ensemble du réseau hydrographique. Aujourd'hui encore, certains types d'aménagement ne sont pas compatibles avec une gestion piscicole optimale. Une stratégie globale est difficile à envisager, mais quelques recommandations concernant la gestion du corridor végétal des rivières peuvent être proposées.

Mots-clés : Formations végétales arborées, hydrosystème, habitat piscicole, chaîne trophique, débris ligneux grossiers, gestion.

\section{RIPARIAN FOREST OF STREAM AND FISH MANAGEMENT}

\section{SUMMARY}

The riparian forest is in close relation with the aquatic environment. The hydrodynamic conditions govern the repartition of vegetal communities. Inversely, the riparian vegetation is a hydrosystem structural element which influences the hydrodynamics. Its single presence on the streambank or indirectly its ability to produce, export and retain coarse woody debris complexifies the aquatic biotope, creates a diversified environment which is very interesting for the biological and thus fishes communities. It also governs the 
trophic chain in regulating nutrian deposits and temperature, in limiting autotrophic production (shade), in bringing in organic debris. Aquatic and terrestrial environments are very interconnected ; consequently, it is necessary to study and better understand the biostructural dynamics of the riparian forest in order to include it in the management of any kind of hydrosystems. Today, a lot of planning technics and projects are not in agreement with a satisfying management of fisheries. A global strategy of riparian forest management is still untimely; few recommendations can be only proposed.

Key-words : Riparian forest, hydrosystem, fish habitat, trophic chain, coarse woody debris, riparian management.

\section{INTRODUCTION}

Les formations végétales arborées riveraines des cours d'eau qui occupent naturellement le lit majeur des rivières sont sous l'influence de mécanismes complexes et originaux. A l'interface entre les milieux terrestres et aquatiques, elles disposent d'une dynamique propre et peuvent se définir comme un écotone. Leur extension, suivant l'ordre du cours d'eau, peut varier de quelques mètres (ripisylve définie par CEMAGREF, 1988) à plusieurs centaines de mètres (forêt de plaine d'inondation ou communément appelée forêt alluviale). Le fonctionnement des écotones a largement été abordé par les scientifiques. Un programme MAB-UNESCO «role of land/inland water ecotones in landscape management and restoration" traite de ce sujet (NAIMAN et al., 1989), mais l'application des connaissances scientifiques à la gestion reste délicate.

Dans cet article, nous aborderons le rôle des formations végétales arborées riveraines dans la structuration et le fonctionnement des hydrosystèmes. Leur existence, leur composition floristique et leur extension spatiale sont dépendantes des écoulements superficiels et phréatiques. Inversement, les écosystèmes aquatiques sont dynamiquement dépendants de la zone rivulaire ; les poissons, en fin de chaîne alimentaire, sont souvent utilisés comme des descripteurs de ce fonctionnement (ZALEWSKI et al., 1991). Dans la rivière considérée comme un continuum (VANNOTE et al., 1980), l'influence hydromorphologique et biologique des formations végétales arborées riveraines varie selon l'ordre du cours d'eau et leur position le long de ce continuum (CUMMINS et al., 1983). La végétation sera d'autant plus influente que le cours d'eau est de faible largeur. Aux difficultés de compréhension liées à cette complexité spatiodynamique s'ajoute la rareté de systèmes de référence. Les études historiques indiquent qu'une grande partie de l'intégrité biologique des cours d'eau a été perdue il y a plusieurs siècles en Europe et en Asie, et seulement un siècle en Amérique du Nord (HARMON et al., 1986).

En France, depuis 1945, suite au bouleversement du monde rural, l'entretien des cours d'eau non domaniaux a été progressivement délaissé. Leur retour vers un état d'équilibre dynamique (réajustements morphodynamique et biodynamique) est devenu rapidement gênant pour certaines activités humaines (agriculture, tourisme...). La réponse a d'abord été brutale, consistant à transformer les rivières en ouvrages techniques aptes à évacuer les crues. Actuellement, des stratégies d'aménagement plus souples intégrant d'autres éléments structurels de l'écosystème sont prônées (restauration et entretien de la végétation riveraine par les techniques dites de génie biologique). Cependant, ces approches nouvelles, visant le plus souvent à stabiliser le système, intègrent-elles bien les connaissances récentes du fonctionnement naturel des cours d'eau?

Par ailleurs, comment une gestion des espaces arborés riverains, sans doute différente de celle préconisée aujourd'hui, peut-elle conduire à une valorisation de la ressource piscicole?

Cet article propose une synthèse bibliographique concernant le rôle des formations végétales arborées riveraines dans les écosystèmes d'eau courante.

La végétation riveraine sera d'abord perçue comme un élément structurel, puis comme une source de diversification et de stabilisation des habitats piscicoles, et enfin comme un élément contrôlant le fonctionnement biologique. En conclusion, des stratégies de gestion seront esquissées. 


\section{1) STRUCTURE BIOMORPHOLOGIQUE}

Les communautés biologiques de l'hydrosystème sont en équilibre dynamique (SCHUMM, 1977) avec les conditions physiques. II existe un ajustement permanent et interactif des différents éléments structurels biotiques (végétaux) et abiotiques (hydrologie, morphologie).

L'eau dans sa dynamique spatiotemporelle régit le fonctionnement de ce système (AMOROS et al., 1982). La morphologie s'ajuste aux conditions hydrologiques (SCHUMM, 1977 ) ; les conditions hydromorphodynamiques dictent la distribution des plantes, la répartition des communautés forestières (CARBIENER, 1970 ; NANSON, 1977 ; HUPP \& OSTERKAMP, 1985 ; PAUTOU \& DÉCAMPS, 1985).

Réciproquement, les formations végétales arborées riveraines peuvent constituer elles-mêmes un élément structurel de l'écosystème et agir sur l'hydraulique et la morphologie (Fig. 1). La dynamique de la végétation riveraine et des débris ligneux grossiers est étudiée successivement.
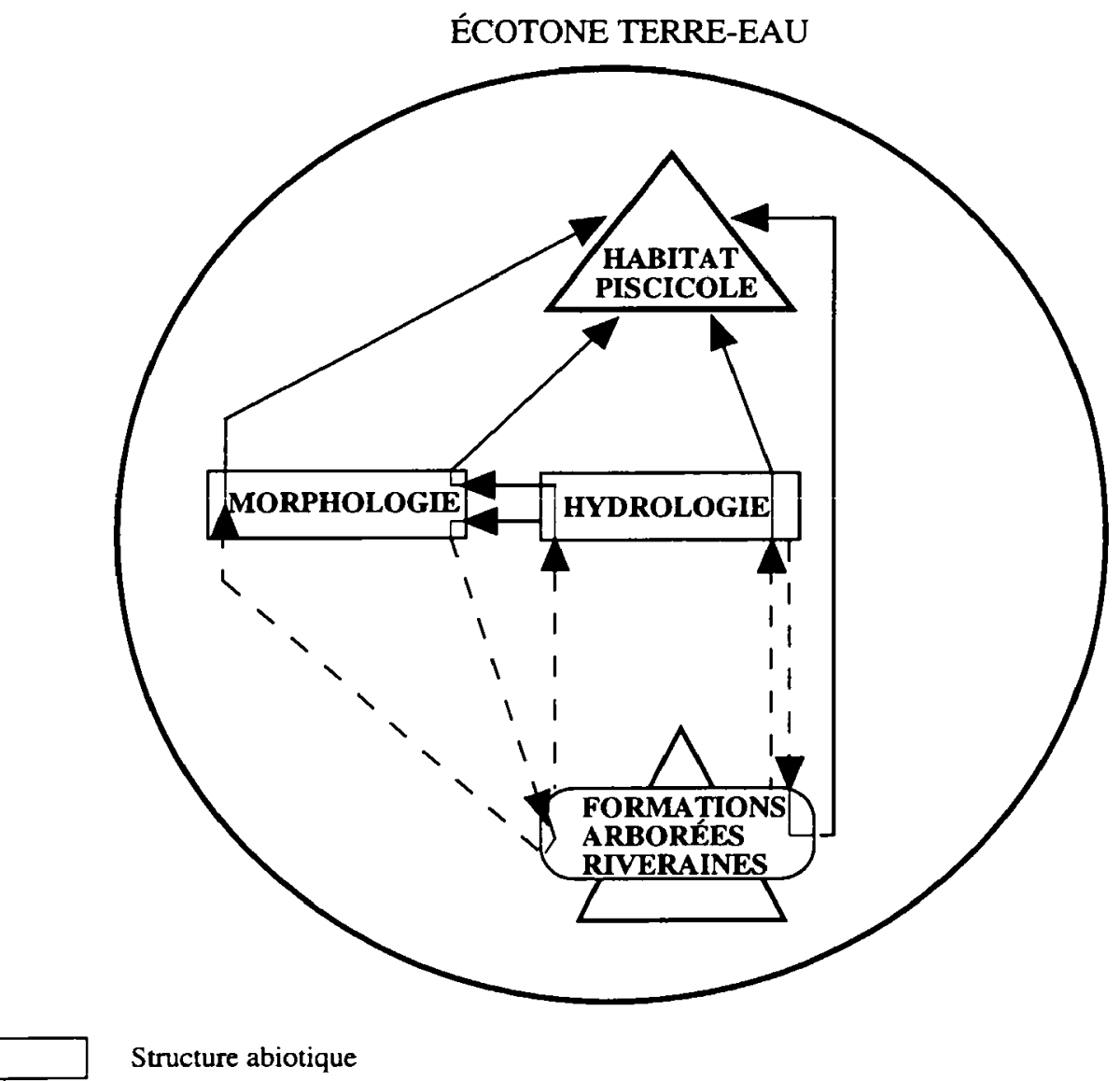

Structure abiotique

Structure biotique

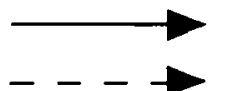

Création et diversification (dynamique fonctionnelle)

"Cellule fonctionnelle"

Dynamique d'ajustement des paramètres structurels

Figure 1: La ripisylve, un élément biostructurel créateur de l'habitat.

Figure 1: The riparian forest as a biostructural component of habitat. 


\section{Potentialités structurelles de la végétation}

Les formations végétales arborées riveraines exercent un contrôle sur les conditions physiques de l'environnement aquatique. La résistance de la végétation à l'écoulement est un mécanisme reconnu (KOUWEN \& RUH-MING, 1980) mais difficile à modéliser, sa flexibilité et sa densité variant sans fin. Certains auteurs ont abordé l'influence de la végétation du bassin versant sur l'hydrologie (HARR et al., 1982), d'autres de la végétation rivulaire sur la sédimentologie (DAHLSKOG, 1966 ; NANSON \& BEACH, 1977 ; LYNCH et al., 1977 ; WITT, 1985) et la morphologie (HADLEY, 1961 ; HICKIN, 1984 ; GREGORY \& GURNELL, 1988 ; CLIFTON, 1989). La plupart de ces études portent sur des rivières de rang 1 à 3 (d'après la méthode de STRAHLER, 1957) situées dans des régions topographiquement et climatiquement très différentes. La végétation de berge influe d'autant plus sur la morphologie et l'hydraulique du cours d'eau que celui-ci est de faible largeur.

Les racines stabilisent la berge, retardent l'érosion et favorisent localement des encorbellements (SMITH, 1976 ; HICKIN \& NANSON 1984 ; MEEHAN et al., 1977 ; BERGERON \& ROY, 1985). Les propriétés de la végétation ripariale favorisant la stabilité de la berge, et donc la pérennisation d'un état de la rivière, sont de plus en plus utilisées dans les aménagements souples de génie biologique (DETHIOUX, 1988 ; LACHAT, 1991 ; PETERSEN et al., 1992). L'aptitude à stabiliser la berge dépend des espèces. LACHAT (1991) a recensé 48 espèces arborées et arbustives présentes dans les forêts d'inondation de France et de Suisse susceptibles de participer activement à la stabilisation des berges. Chacune dispose d'exigences écologiques et d'une répartition spatiale particulières. II cite, par exemple, Acer pseudoplatanus, Acer platanoides et Fraxinus excelsior sur des berges relativement sèches et à granulométrie sableuse à sablo-limoneuse. Sur des sols plus humides, ils sont remplacés par Alnus glutinosa ou Alnus incana dans les régions alpines sur des sols limono-argileux et différents saules (Salix alba, Salix viminalis ou Salix eleagnos) sur des sols graveleux.

\section{Potentialités structurelles des débris ligneux grossiers}

Si la connaissance des formations végétales arborées riveraines et surtout celle de leur rôle stabilisateur des berges progressent en Europe, leur influence indirecte (production et consommation de débris ligneux grossiers) est encore méconnue du fait de l'ancienneté de la pression anthropique. Trois publications majeures (HARMON et al., 1986 ; BISSON et al., 1987 ; MASER et al., 1988) proposent une solide synthèse des travaux récemment entrepris sur ce thème. Ils montrent que les débris ligneux grossiers ont été largement présents dans les cours d'eau originels et qu'ils induisent des processus morphologiques originaux qui diffèrent selon l'ordre des cours d'eau.

\section{a) Approche historique et géographique}

Certaines études extra-européennes montrent que les rivières originelles, qu'elles soient rapides ou lentes, ont un chenal largement occupé par le bois mort. Des barrages de débris organiques sont décrits sur l'Amazone, le Congo, l'Orénoque, le Gange, le Mississippi, le Mackenzie, en Chine (LYELL, 1969 ; HARMON et al., 1986). Au siècle dernier, les cours d'eau américains du Nord-Ouest (SEDELL \& LUCHESSA, 1982 ; SEDELL \& FROGGATT, 1984 ; TRISKA, 1984) ou des plaines subtropicales du Sud (WALLACE \& BENKE, 1984) étaient obstrués de barrages organiques.

Aujourd'hui, rares sont les rivières recelant d'importantes quantités ligneuses. La masse totale des débris ligneux grossiers dans les écosystèmes fluviaux tempérés varie considérablement et peut atteindre dans des rivières peu perturbées drainant certaines vieilles forêts de conifères du Nord-Ouest américain 1800 tha (HARMON et al., 1986) à 2300 tha (SEDELL et al., 1988).

Les études récentes restent encore limitées à quelques régions et à des cours d'eau souvent d'ordre 1 à 6, c'est-à-dire faible à moyen. HARMON et al. (1986) et BISSON et al. (1987) se focalisent sur les cours d'eau du Nord-Ouest des Etats-Unis où le milieu très forestier et parfois relativement épargné par l'activité forestière est encore riche en bois mort. D'autres auteurs américains s'intéressent aux rivières de plaine côtière subtropicale du Sud-Est (WALLACE \& BENKE, 1984 ; BENKE et al., 1985) ou aux petits cours d'eau du 
Nord-Est (ZIMMERMAN et al., 1967 ; BILBY, 1979 et 1981). Les études européennes (GREGORY en Angleterre, 1992 ; PIÉGAY \& BRAVARD, en France, sous presse, 1993) ou néozélandaises (MOSLEY, 1981) restent encore rares.

\section{b) Processus spécifiques à certains hydrosystèmes}

Les structures biomorphologiques diffèrent d'un hydrosystème à l'autre. Les volumes, la fréquence, la forme, la localisation et l'aire d'influence des débris ligneux grossiers sont régis par la taille du cours d'eau, la morphologie de la vallée, les facteurs à l'origine des débris dans le chenal (KELLER \& SWANSON, 1979 ; KELLER \& MACDONALD, 1983), mais aussi par l'âge et la dynamique de régénération de la forêt d'inondation. Plus le cours d'eau est important, plus ses eaux sont aptes à exporter ce matériel vers l'aval ou dans la plaine d'inondation (SWANSON \& LIENKAEMPER, 1978 ; TRISKA et al., 1982 ; SEDELL \& LUCHESSA, 1982 ; CUMMINS et al., 1983). Les volumes de débris ligneux grossiers relatifs à la taille du cours d'eau sont d'autant plus faibles que le rang du cours d'eau augmente (ANDERSON et al., 1978 ; BILBY, 1979 ; LIENKAEMPER \& SWANSON, 1986). Dans les cours d'eau petits et moyens, les débris ligneux grossiers localisés au hasard forment de larges accumulations à structure très ouverte (HARMON et al., 1986), alors que dans les grands cours d'eau ils s'accumulent en embâcles dans des sites préférentiels tels que la partie amont des bancs de convexité ou des chenaux secondaires, la concavité des méandres... (KELLER \& SWANSON, 1979 ; SWANSON \& LIENKAEMPER, 1982 ; PIÉGAY, 1993).

Actuellement, l'étude des débris ligneux grossiers n'a été entreprise que dans les petits cours d'eau de montagne drainant une forêt de conifères pluricentenaires ou les cours d'eau intermédiaires de plaine à méandres actifs (Squamish canadienne, Ain français) ou à anastomoses (Willamette et Red River des Etats-Unis).

Dans les premiers, les débris ligneux grossiers modifient leur géométrie, ils contrôlent le transit de la charge de fond (MOSLEY, 1981 ; MARSTON, 1982 ; KELLER \& MACDONALD, 1983 ; GREGORY, 1992), modifient la pente longitudinale (HEEDE, 1972 ; KELLER \& SWANSON, 1979), augmentent la largeur du chenal (ZIMMERMAN et al., 1967 ; GURNELL \& GREGORY, 1984) et le nombre de mouilles (GRETTE, 1982 ; HEIFETZ et al., 1986 ; O'CONNOR \& ZIEMER, 1989 ; ROBISON \& BESCHTA, 1990). les débris ligneux grossiers dissipent l'énergie des eaux courantes (ROBISON, 1987 ; SEDELL et al., 1988) et contrôlent l'écoulement dans le chenal (HEEDE, 1985 ; LISLE, 1982 ; GREGORY et al., 1985). Ils peuvent augmenter la stabilité de l'hydrosystème (SWANSON \& LIENKAEMPER, 1978 ; KELLER \& TALLY, 1979 ; TOEWS \& MOORE, 1982). Par contre, en Angleterre dans des régions de collines plus récemment recolonisées par la forêt, les barrages organiques beaucoup moins stables peuvent générer une plus grande instabilité du lit (GREGORY et al., 1985 ; GREGORY, 1992).

Quant aux grands cours d'eau tempérés de piémont, ils forment à l'origine un réseau de radiers, d'îles, de mouilles et de barrages organiques sans chenal principal et continuellement ombragé (SEDELL et al., 1988). Les débris ligneux grossiers favorisent l'érosion de berges et la diversification des géoformes (KELLER \& SWANSON, 1979 ; HOUSE \& BOEHNE, 1986) et augmentent donc les connexions lit majeur - lit mineur. Ils peuvent aussi stabiliser et protéger des sites qui, une fois établis, permettent le développement végétal. Les rives sont stabilisées alors par les racines (SWANSON \& LIENKAEMPER, 1982). De même, les arbres tombés mais encore accrochés par les racines aux rives, voire ensevelis dans la rive, retardent localement l'érosion. Le castor est un agent morphogène et biogène étroitement intégré à cette dynamique ; il joue un rôle prépondérant dans la diversification du milieu (JOHNSTON \& NAIMAN, 1987 ; NAIMAN et al., 1988).

\section{II) CRÉATION ET DIVERSIFICATION DE L'HABITAT PISCICOLE}

La diversité et la productivité piscicoles semblent d'autant plus élevées que les habitats sont diversifiés, réduisant ainsi la compétition intra et interspécifique (GORMAN \& KARR, 1978 ; BISSON et al., 1981). 


\section{Rôle de la végétation riveraine}

\section{a) à l'échelle du lit majeur}

Les formations végétales arborées riveraines favorisent la diversité des habitats. SIOLI (1982), NAIMAN \& DÉCAMPS (1990) notent que la connexion plaine alluviale-chenal principal est importante pour la migration des poissons non seulement à des fins trophiques mais également reproductives. SWANSON \& LIENKAEMPER (1982) expliquent que les processus géomorphologiques créent des habitats aquatiques distincts dans la vallée. Ils distinguent sur la South Fork Hoh River (cours d'eau de rang intermédiaire) chenal principal, bras morts, tributaires phréatiques et tributaires de versant dans lesquels I'hydrodynamique et l'influence de la végétation sont très distinctes. AMOROS \& ROUX (1988), MOORE \& GREGORY (1989), COPP et al. (1992) montrent l'importance des habitats latéraux pour les juvéniles de salmonidés. Spotts (1989) explique que la destruction de $98 \%$ de la ripisylve de la Sacramento River entre 1848 et 1990 aurait induit une réduction de $80 \%$ des poissons en liaison avec la perte de leur habitat et l'allongement de la liste d'espèces menacées.

La régénération des formations végétales arborées riveraines associée à l'érosion latérale maintient un écosystème productif. Sur le cours moyen du Sacramento, l'érosion des berges favorise les aires de ponte pour les salmonidés telles que des seuils de convexité, des chenaux de recoupement de méandres et des aires à chenaux multiples (BUER et al., 1989). Ainsi, le rétablissement du méandrage et donc de l'érosion latérale sur les rivières rectifiées permet de retrouver la séquence seuil-mouille et de recréer la diversité de l'habitat (PETERSEN et al., 1992).

\section{b) à l'échelle de la berge}

La ripisylve influence l'habitat des salmonidés en favorisant les couverts, soit directement en créant des encorbellements, soit en étant la source de débris ligneux grossiers (BOUSSU, 1954 ; MEEHAN et al., 1977 ; HOUSE \& BOEHNE, 1986 ; HUNTER, 1991). Ces formations jouent un rôle d'abri face à des conditions climatiques et hydrologiques difficiles (BISSON et al., 1987 ; POUJARDIEU, 1988). Les racines sont bien sûr des caches, mais aussi des supports de ponte pour les poissons (G.I.R.E.A., 1987).

Dans les cours d'eau de faible énergie, le mouvement et le dépôt de sédiments, suite à une modification de la végétation du bassin versant et à une dégradation de la ripisylve, provoquent la destruction des habitats. Absente, la végétation rivulaire (herbacées, système racinaire) ne filtre plus les sédiments fins en transit. La charge en suspension affecte la vie piscicole (séquelles au niveau des branchies, réduction des capacités visuelles). La sédimentation récente réduit l'écoulement intragraveleux, limite les apports en oxygène nécessaire à l'incubation des oeufs et des alevins et favorise des dommages par abrasion physique (MEEHAN et al., 1977 ; LYNCH et al., 1977 ; GREGORY \& STOKOE, 1981 ; MORING et al., 1985 ; CAMPBELL \& DOEG, 1989). Dans ce cas, par sa présence, la ripisylve réduira la charge sédimentaire par filtration.

La ripisylve agit également directement sur l'éthologie des poissons en créant une protection contre des prédateurs potentiels (MEEHAN et al., 1977, 1987 ; G.I.R.E.A., 1987). En effet, l'ombre créée par la végétation arborée peut dissimuler les poissons qui possèdent un comportement territorial, et ainsi diminuer l'agressivité vis-à-vis de congénères. De nombreux auteurs ont constaté chez un grand nombre d'espèces de salmonidés, un préférendum en période de repos pour des secteurs ombragés (BRUSVEN et al., 1986 ; MEEHAN et al., 1987). Par contre, la recherche de nourriture et l'alimentation sont facilitées dans les secteurs à canopée ouverte. WILZBACH et al. (1986) trouvent chez les truites une corrélation positive entre le pourcentage de proies capturées et la surface éclairée. La plus grande visibilité des proies en secteur éclairé et donc de l'efficacité de capture agit sur la croissance des truites. La disponibilité des proies est un facteur important qui détermine la distribution et l'abondance des truites (WILZBACH, 1985 ; WILZBACH \& HALL, 1985). 


\section{Le bois mort, un agent de diversification et de stabilisation des habitats}

Pour beaucoup d'organismes, les débris ligneux grossiers et les microenvironnements qui leur sont associés représentent un habitat (HARMON et al., 1986 ; GREGORY \& DAVIS, 1992 ; O'CONNOR, 1992). Certains en sont strictement dépendants, d'autres bénéficient de leur opportunité.

La perception du rôle des débris ligneux grossiers dans les hydrosystèmes a beaucoup changé depuis 1970 (SEDELL et al., 1985). Auparavant, ils étaient perçus sur certains types de cours d'eau comme une cause de la réduction de l'oxygène dans l'eau (HARMON et al., 1986) et un obstacle à la migration des salmonidés retournant pondre dans les cours amont (MERRELL, 1951 ; HOLMAN \& EVANS, 1964). Cet impact est aujourd'hui reconnu comme étant négligeable (SEDELL \& LUCHESSA, 1982 ; BRYANT, 1983). Les débris ligneux augmentent la qualité de l'habitat piscicole en complexifiant et diversifiant le milieu (GARD, 1961 ; LISLE, 1982 ; FRANKLIN, 1988 ; FAUSCH \& NORTHCOTE, 1992).

Dans un cours d'eau de rang 5 (Fish Creek) d'Orégon, $44 \%$ de l'habitat piscicole est influencé par la présence de débris ligneux grossiers (EVEREST et al., 1988). L'abondance piscicole autour des débris ligneux grossiers augmente avec la complexité croissante de l'accumulation (FORWARD, 1984). Dans certaines grandes rivières, la densité de salmonidés et d'invertébrés est la plus importante dans les secteurs où s'accumulent les débris ligneux grossiers (WARD et al., 1982 ; SEDELL et al., 1984). Ainsi la stabilité et la longévité des débris ligneux grossiers influencent fortement la qualité de l'habitat en maintenant sa diversité quelle que soit la taille du cours d'eau (BISSON et al., 1987 ; SEDELL et al., 1988). La lente décomposition du bois en milieu aquatique renforce sa capacité à maintenir des habitats (HARMON et al., 1986).

Le petit cours d'eau de montagne drainant une vieille forêt de conifères où les débris ligneux grossiers influencent les caractères biologiques et physiques semble être l'habitat salmonicole le plus productif (SWANSON \& LIENKAEMPER, 1978 ; SEDELL et al., 1988). Les escaliers de troncs augmentent la diversité des habitats en permettant une succession longitudinale mouilles - seuils (BISSON et al., 1987 ; SEDELL \& LUCHESSA, 1982 ; SEDELL et al., 1987). Sur des cours d'eau à forte granulométrie, de gros blocs peuvent jouer un rôle identique. Par contre, sur des cours d'eau à fond sableux, seules des grosses structures ligneuses peuvent générer ce type de faciès. Les mouilles profondes disposant d'un couvert offrent aux organismes une meilleure chance d'échapper aux prédateurs terrestres (BUGERT et al.,1991) et permettent la coexistence d'espèces ou d'individus d'âges différents dans la colonne d'eau (SEDELL et al., 1988 ; BILBY \& WARD, 1989). Elles fournissent un site de repos pour la plupart des poissons (Oncorhynchus kisutch, Salvelinus malma, Oncorhynchus mykiss) en toute saison, surtout en période de crue hivernale ou de migration (BUSTARD \& NARVER, 1975 ; BISSON et al., 1987 ; MURPHY et al., 1984 ; SEDELL et al., 1988). En période d'étiage, elles retiennent des quantités d'eau importantes, et en raison de leur profondeur, conservent une eau plus fraîche, servant de refuges thermiques aux poissons durant les étés chauds (BISSON et al., 1987). Par contre, les mouilles sans couvert ne sont pas occupées (HEIFETZ et al., 1986). Ainsi, une réduction des débris peut réduire le nombre d'individus quelle que soit l'espèce de salmonidés (MORTENSEN, 1977 ; TSCHAPLINSKI \& HARTMAN, 1983 ; ANGERMEIER \& KARR, 1984 ; BRYANT, 1981 et 1985 ; DOLLOFF, 1986 ; BISSON et al., 1987).

Comme l'ombre, les débris ligneux grossiers peuvent jouer le rôle de barrières visuelles, diminuant l'agressivité interspécifique mais également intraspécifique entre différents stades de développement (ELLIOTT, 1986), permettant ainsi une bonne croissance des individus. BOUSSU (1954) montre qu'une couverture de débris artificiels peut conduire à augmenter le nombre et la taille des truites.

\section{III) FORMATIONS ARBORÉES RIVERAINES ET FONCTIONNEMENT BIOLOGIQUE}

Dans les cours d'eau de rangs inférieurs et moyens, les formations végétales arborées riveraines contrôlent la chaîne trophique à sa base en injectant dans le système des détritus organiques, en filtrant les apports en nutriments, en limitant la production 
autotrophe par ombrage et en régulant la température (Fig. 2). Ainsi, toute atteinte à la végétation induira des changements au niveau de la chaîne trophique à l'intérieur du cours d'eau et donc sur la productivité piscicole (BILBY \& BISSON, 1992).

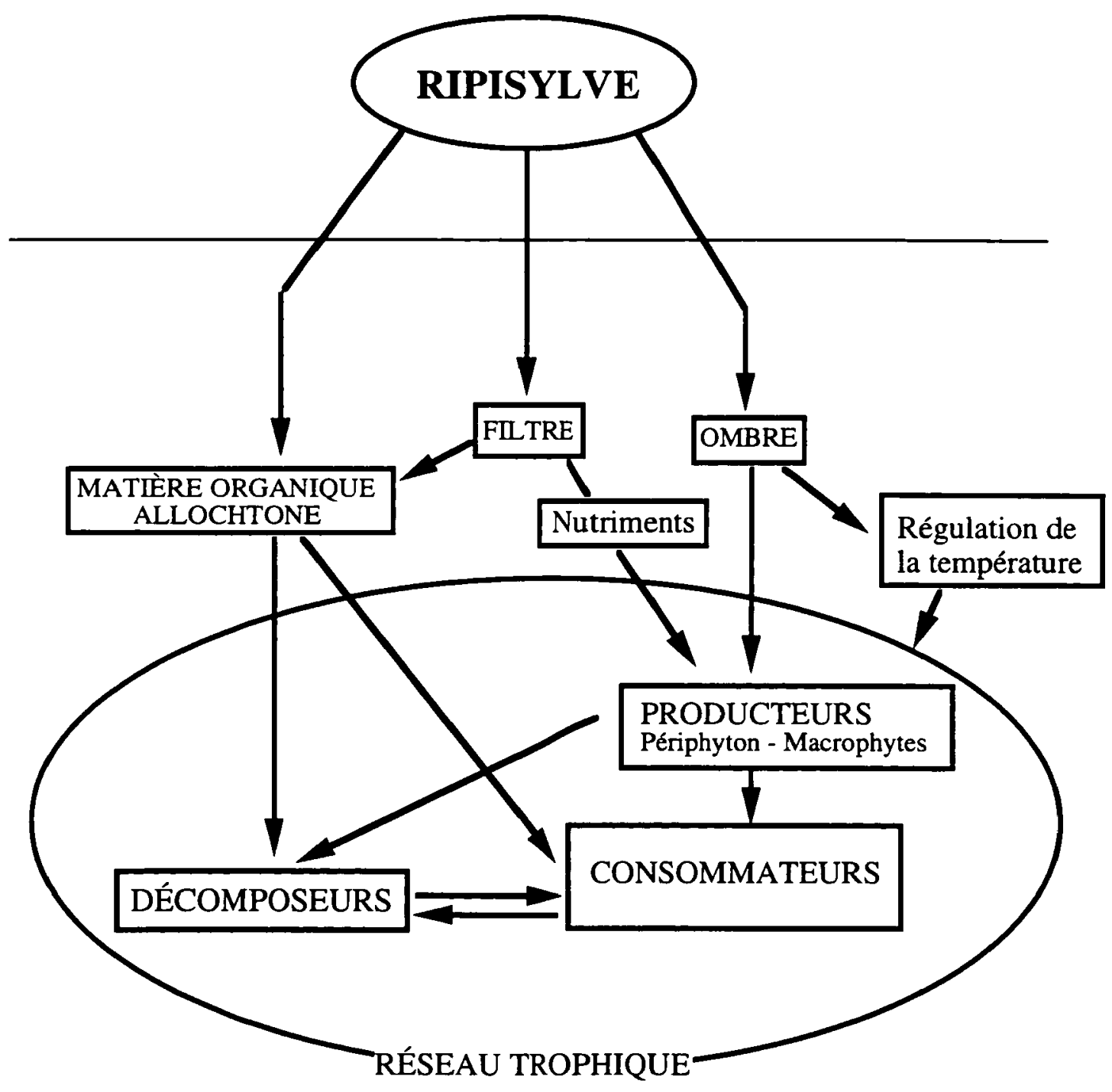

Figure 2 : Influence de la ripisylve sur les processus trophiques dans les écosystèmes d'eau courante.

Figure 2 : Influence of riparian forest on trophic processes in running water systems.

\section{Canopée de la végétation de berge}

\section{a) Rôle de l'ombre et de la lumière}

La lumière et la vitesse du courant sont des facteurs limitants du développement périphytique ou macrophytique ; la quantité de nutriments (azote, phosphore) selon leur teneur agira seulement sur la trophie du système (HAURY, 1985). Ainsi, une coupe à blanc agit nettement sur la production primaire et donc sur le rapport autotrophes/hétérotrophes. A court terme, un milieu ouvert est globalement plus productif qu'un milieu fermé. II engendre une production autotrophe plus importante (HAWKINS et al., 1983 ; BEHMER \& HAWKINS, 1986), d'autant plus que l'effet filtre des nutriments aura disparu avec le 
système racinaire (HOLOPAINEN et al., 1991). Or, le périphyton possède une qualité alimentaire plus élevée par rapport aux litières de feuilles mortes ou de branchages (HAWKINS et al.,1982 ; STEINMAN et al., 1988). Ces modifications induiront donc des changements au niveau des groupes trophiques supérieurs (macroinvertébrés, poissons). Dans des rivières de faible énergie, cet état peut engendrer une eutrophisation du milieu néfaste à la vie des organismes. Dans d'autres cours d'eau à dynamique plus forte, l'augmentation de la production primaire, suite à un éclaircissement de la végétation rivulaire, engendre une augmentation de l'abondance des invertébrés et de ce fait des prédateurs incluant les poissons (MURPHY et al., 1981 et 1984).

L'éclairement influence également la composition périphytique. En site ombragé, le peuplement est dominé par les diatomées et, en site plus ouvert, ce sont les algues filamenteuses et d'autres macroalgues qui dominent (CAMPBELL \& DOEG, 1989 ; HICKS et al., 1991 ; MURPHY et al., 1981).

La ripisylve régule également la production macrophytique (G.I.R.E.A., 1987 ; CEMAGREF Bordeaux, 1988 ; POUJARDIEU, 1988 ; GREGORY et al., 1989). Suivant la largeur du cours d'eau, l'ombre créée par une végétation arborée rivulaire peut agir sur la biomasse et le développement des plantes aquatiques (HAURY, 1985). Lorsque les macrophytes deviennent trop abondants, la création de zones d'ombre peut être une réponse au problème de gestion de la biomasse des plantes aquatiques (DAWSON \& KERN-HANSEN, 1979 ; DAWSON \& HASLAM, 1983).

\section{b) Modification de la température}

La végétation rivulaire contrôle directement la température de l'eau qui est proportionnelle à la surface exposée aux radiations et à l'énergie solaire entrante. L'impact de la ripisylve sur la température de l'eau varie suivant la morphologie du cours d'eau et le port des végétaux rivulaires. Ainsi, sur les cours d'eau larges (ordre 6 à 7), seuls les arbres pourront fournir un ombrage efficace en bordure de berges alors que, sur les cours d'eau moyens (ordre 3 à 5), des taillis suffiront à modérer la température de l'eau (PLATTS, 1991). L'enlèvement de la végétation riveraine sur des petits cours d'eau non montagneux peut provoquer une augmentation des températures estivales (de 3 à $5^{\circ} \mathrm{C}$ ), une diminution des températures hivernales (de 1 à $2^{\circ} \mathrm{C}$ ) et engendrer des variations quotidiennes plus prononcées (CLEMENT, 1985 ; PLATTS \& NELSON, 1989).

Ces changements de régime thermique modifient les communautés benthiques en agissant directement sur le cycle vital des organismes (QUINN et al., 1992) et indirectement sur la qualité nutritive de la nourriture disponible (FULLER \& FRY, 1991). Une variation de quelques degrés peut agir directement sur la fécondité ou la taille des individus et indirectement sur la qualité nutritive des aliments en contrôlant, par exemple, le taux de colonisation des feuilles par les microorganismes. Ainsi, l'abondance et la diversité des communautés benthiques sont affectées. Une augmentation de température favorisera le développement de la microflore qui utilise la matière organique; ce qui va provoquer une décomposition plus rapide et une augmentation de la production d'invertébrés (BESCHTA et al., 1987). Mais ceci n'est qu'un effet transitoire qui accélère la consommation des stocks en matière organique et, à long terme, on assiste à une diminution de la disponibilité en matériel organique.

Comme pour les communautés benthiques, la température agit sur le groupe fonctionnel des prédateurs supérieurs. Par leur position dans la chaîne trophique, ils intègrent toute modification trophique du système. De plus, la température intervient directement sur leur cycle de développement et leur métabolisme. Par exemple, la végétation rivulaire permettra de maintenir une température propice au développement, à la reproduction, à l'alimentation des populations résidentes de salmonidés (MEEHAN et al., 1977). CRISP (1989) donne pour différentes espèces de truites un optimum entre 4 et $19^{\circ} \mathrm{C}$, et une température de $23^{\circ} \mathrm{C}$ comme critique pour un grand nombre de salmonidés. Une température élevée peut provoquer une augmentation des affections virales ou bactériennes, augmenter la mortalité par maladie (BESCHTA et al., 1987). La présence le long de cours d'eau d'ordre moyen de secteurs à l'ombre peut abaisser la température de quelques degrés. Selon HALL \& LANTZ (1969), en été, sous un climat tempéré, un secteur de $200 \mathrm{~m}$ ombragé entraîne une diminution de la température de l'eau de $3^{\circ} \mathrm{C}$. 
Indirectement, une augmentation de température agira sur les organismes en diminuant la solubilité de l'oxygène dans l'eau (HALL \& LANTZ, 1969).

\section{Les formations végétales arborées riveraines et la dynamique des éléments nutritifs}

Proches du cours d'eau, les formations végétales arborées riveraines agissent directement comme source de matière organique allochtone (feuilles, tiges, débris ligneux grossiers...) ; plus éloignées, par le jeu du «spiralling» ou parcours en hélice des nutriments (DÉCAMPS \& NAIMAN, 1989), elles agiront sur le stockage, le recyclage ou le relargage vers l'aval des éléments nutritifs (effet filtre). La qualité de ces apports allochtones variera avec la nature des espèces constitutives de la végétation arborée. Par exemple, des aiguilles de résineux seront beaucoup moins dégradables que des feuilles plus tendres d'aulnes ou de saules. De même, le conditionnement de ces apports par les microorganismes sera fortement dépendant de leur origine (CHERGUI, 1988). La nature des espèces arborées agira donc sur la densité des peuplements d'invertébrés aquatiques, notamment des déchiqueteurs de matière organique.

A l'intérieur du cours d'eau, les embâcles servent de pièges potentiels et de structures de rétention des particules détritiques telles que les feuilles, les tiges (BRYANT, 1981 ; PROCHAZKA \& STEWART, 1991). BILBY \& LIKENS (1980) montrent sur des bassins versants boisés (forêt non perturbée âgée de 65 ans) que les embâcles dans des cours d'eau de premier ordre piègent $75 \%$ de la matière organique, dans des cours d'eau de second ordre $58 \%$, et $20 \%$ dans des cours d'eau de troisième ordre.

Cet amas de débris organiques sert de nourriture, de site de ponte, de croissance, de repos et de refuge pour la communauté benthique (MEEHAN et al., 1977 ; ANDERSON et al., 1978 ; TRISKA \& CROMACK, 1980 ; ANGERMEIER \& KARR, 1984 ; WALLACE \& BENKE, 1984). La biomasse d'insectes sur le bois mort est ainsi 20 à 50 fois supérieure à celle d'habitats sableux et 5 à 10 fois supérieure à celle d'habitats vaseux (HARMON et al., 1986). Sur la Satilla, cours d'eau à fond sableux de plaine côtière du Sud-Est de l'État de Géorgie, le bois mort est un habitat potentiellement plus riche que l'habitat benthique tant en qualité (diversité des espèces) qu'en quantité (biomasse). II représente $4 \%$ de la surface totale habitable, mais $60 \%$ de la biomasse (BENKE et al., 1985).

Une diminution de la fréquence des obstacles (débris ligneux grossiers et végétation riveraine arborée) provoque une réduction du temps de rétention et une augmentation de l'exportation vers l'aval des éléments nutritifs (BILBY \& LIKENS, 1980). Dans le cours d'eau, cette modification se traduit par une diminution de la quantité de nourriture assimilable par les populations benthiques qui s'accompagne, avec la disparition de l'effet filtre, d'une augmentation des fines particules néfastes à la vie des organismes. En effet, dans des conditions de température élevée et de faible courant, l'accumulation de ces fines peut engendrer une augmentation de la démande biologique en oxygène (TREMOLIERES \& CARBIENER, 1982 ; HICKS et al., 1991). Ce phénomène, conjointement à une sédimentation accrue, provoque une diminution de la concentration en oxygène à l'intérieur du substrat et une mortalité parmi les alevins à l'émergence et post-émergence (HICKS et al., 1991).

ELLIOTT (1986) constate immédiatement après l'extraction des embâcles du lit mineur une diminution du nombre total d'invertébrés et de la dérive qui subissent une réduction respective de 567 à 200 individus $/ \mathrm{m}^{2}$ et de 28 à 9 individus/échantillon. Après l'enlèvement des embâcles, les poissons peuvent donc être soumis à une diète qui peut provoquer la disparition de certaines classes de tailles très sensibles.

Des modifications des structures de rétentions induiront des perturbations dans la dynamique des éléments nutritifs qui agiront à long terme sur les stocks piscicoles. Dans ce cadre, sur les grands fleuves, DÉCAMPS \& NAIMAN (1989) soulignent l'intérêt du maintien d'une zone riveraine arborée pour la rétention du matériel organique.

Par leur système racinaire, les formations végétales arborées riveraines agissent indirectement sur le cycle des nutriments en jouant le rôle de filtre.

En conditions d'anaérobiose, les microorganismes hétérotrophes, grâce à la synthèse de réductases, réduisent les formes oxydées de l'azote en azote gazeux dispersables dans 
l'atmosphère (PINAY, 1986 ; DÉCAMPS \& NAIMAN, 1989). C'est le principe de la dénitrification biologique, processus majeur dans l'élimination des nitrates. Les milieux humides et boisés, en plus de l'engorgement des sols, offrent des conditions favorables à la réalisation de la dénitrification, en maintenant par ailleurs une teneur généralement élevée en carbone organique (source énergétique pour les microorganismes).

La végétation intervient en stockant provisoirement les nitrates par absorption racinaire. FUSTÉC (1988) a constaté en hiver une remontée des teneurs en nitrates dans les nappes. Ce phénomène est dû au repos végétatif, mais aussi à une augmentation des flux de nitrates indépendante de l'activité racinaire.

En période d'activité végétale, lors du passage sous les formations végétales arborées riveraines, les eaux des nappes se trouvent naturellement épurées des apports en nitrates provenant des zones agricoles riveraines (PETERJOHN \& CORREL, 1984 ; PINAY, 1986). Les travaux réalisés dans la moyenne vallée de la Garonne ont montré que la concentration en nitrates est 20 fois plus faible dans ces zones rivulaires humides que dans les nappes au niveau des plaines agricoles (FUSTEC, 1988). Certains bassins versants sont capables, grâce au maintien de zones humides et boisées, de retenir environ $99 \%$ des nitrates (RHODES et al., 1985). PINAY \& DÉCAMPS (1988) suggèrent qu'une bande rivulaire arborée de $30 \mathrm{~m}$ suffit à éliminer tous les nitrates. PETERSEN et al. (1992) montrent que dès les 10 premiers mètres $90 \%$ des nitrates disparaissent.

Les zones humides et les boisements riverains peuvent piéger, en plus des nitrates, le phosphore et certains constituants toxiques (FUSTEC, 1988). Dans les hydrosystèmes étudiés par PETERJOHN \& CORREL (1984) ou PETTS (1990), environ $90 \%$ de l'azote et $80 \%$ du phosphore sont retenus, utilisés et transformés par les forêts riveraines. Dans les formations arborées, les plantes herbacées peuvent jouer également un rôle prépondérant en filtrant les nutriments (SMITH, 1987 et 1989 ; OSBORNE \& KOVACIC, 1991).

\section{CONCLUSION}

Les peuplements piscicoles sont fortement dépendants des formations végétales arborées riveraines. Celles-ci influencent la diversité et la complexité de l'habitat et contrôlent le fonctionnement biologique (Fig. 3).

Les milieux riverains des cours d'eau, soumis aujourd'hui à une active recolonisation végétale, sont perçus comme des espaces abandonnés et inesthétiques, parfois à risques pour les activités riveraines. Aussi, l'entretien c'est-à-dire le nettoyage rigoureux de la végétation est souvent prôné. Pourtant, une forêt peut vivre sans l'homme sans être vouée à l'anarchie et se perpétuer (WALTER, 1992). De plus, dans les rivières à forte énergie, la biodynamique, source d'instabilité latérale localisée, est un facteur de l'équilibre global et favorise une diversification et une régénération spatiale de l'habitat qui seraient impossibles dans le cadre d'un chenal rectiligne et stable.

Malgré tout, des règles simples de gestion restent difficiles à proposer tant les processus diffèrent d'un hydrosystème à l'autre, dans le temps et au sein même de chacun d'eux. II est souvent difficile de séparer l'effet de tel ou tel facteur. Il arrive même que certains aient des effets antagonistes (JOHNSON \& HEIFETZ, 1985 ; HEIFETZ et al., 1986 ; MURPHY \& MEEHAN, 1991). Par exemple, en été, l'éclaircissement du cours d'eau peut provoquer une augmentation de la production piscicole (essentiellement les jeunes stades) directement reliée à l'abondance du benthos (JOHNSON \& HEIFETZ, 1985). Mais en hiver, cette production chute si l'habitat n'est pas maintenu (embâcle, complexe racinaire en bordure de berge - Fig. 4). En effet, durant les périodes à basse température, le métabolisme des poissons diminue, leur mobilité est réduite, leur survie dépend donc davantage de l'habitat. L'hétérogénéité du milieu semble être la seule solution capable de répondre aux contraintes de vie des différents organismes. Dans un milieu diversifié, tous les stades et tous les groupes trophiques peuvent trouver l'habitat et la nourriture appropriés à leur développement. Les poissons, selon leur stade, peuvent alors migrer dans des secteurs plus favorables.

Dès lors, plusieurs principes de gestion piscicole peuvent être proposés. II paraît nécessaire d'un point de vue conceptuel : 

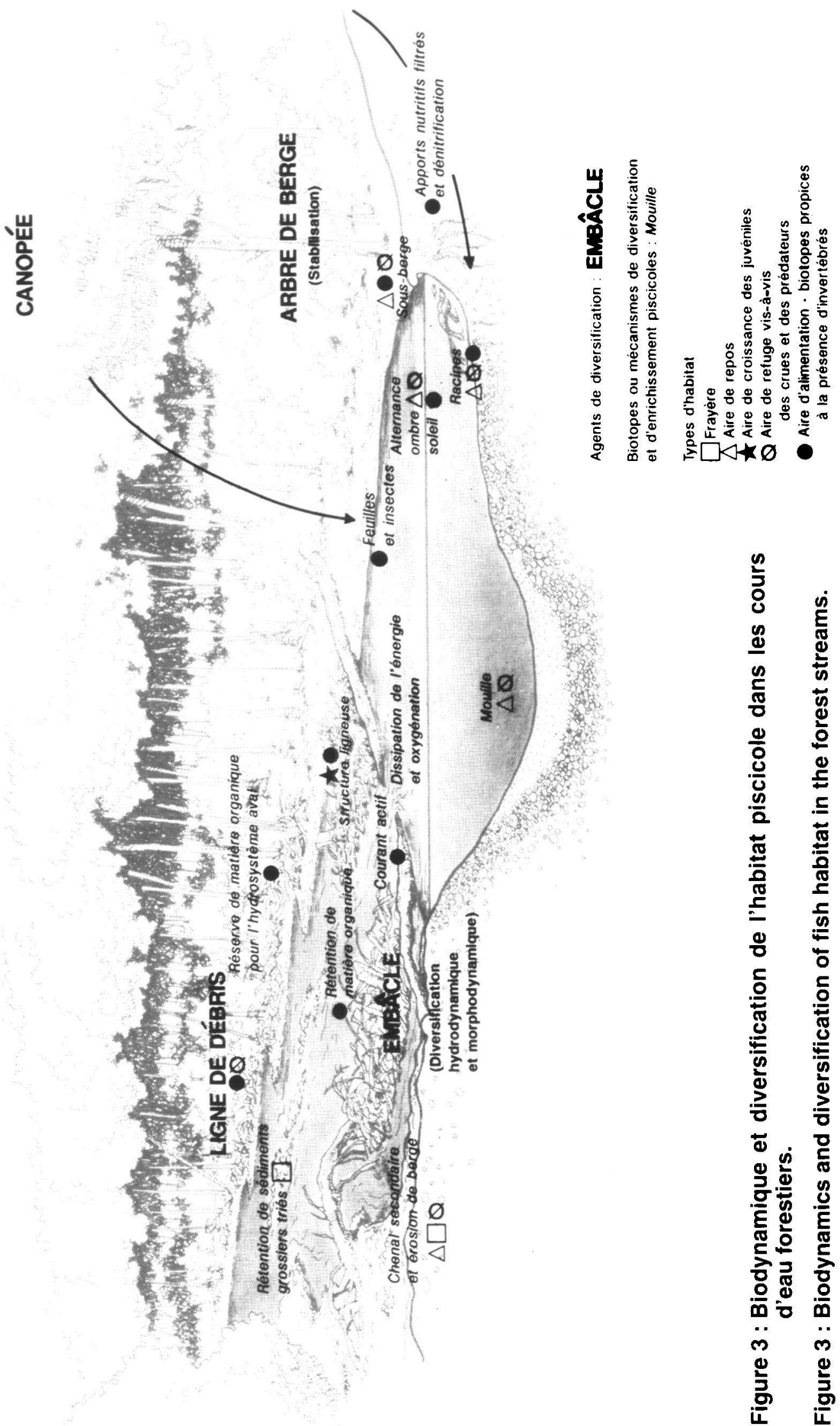


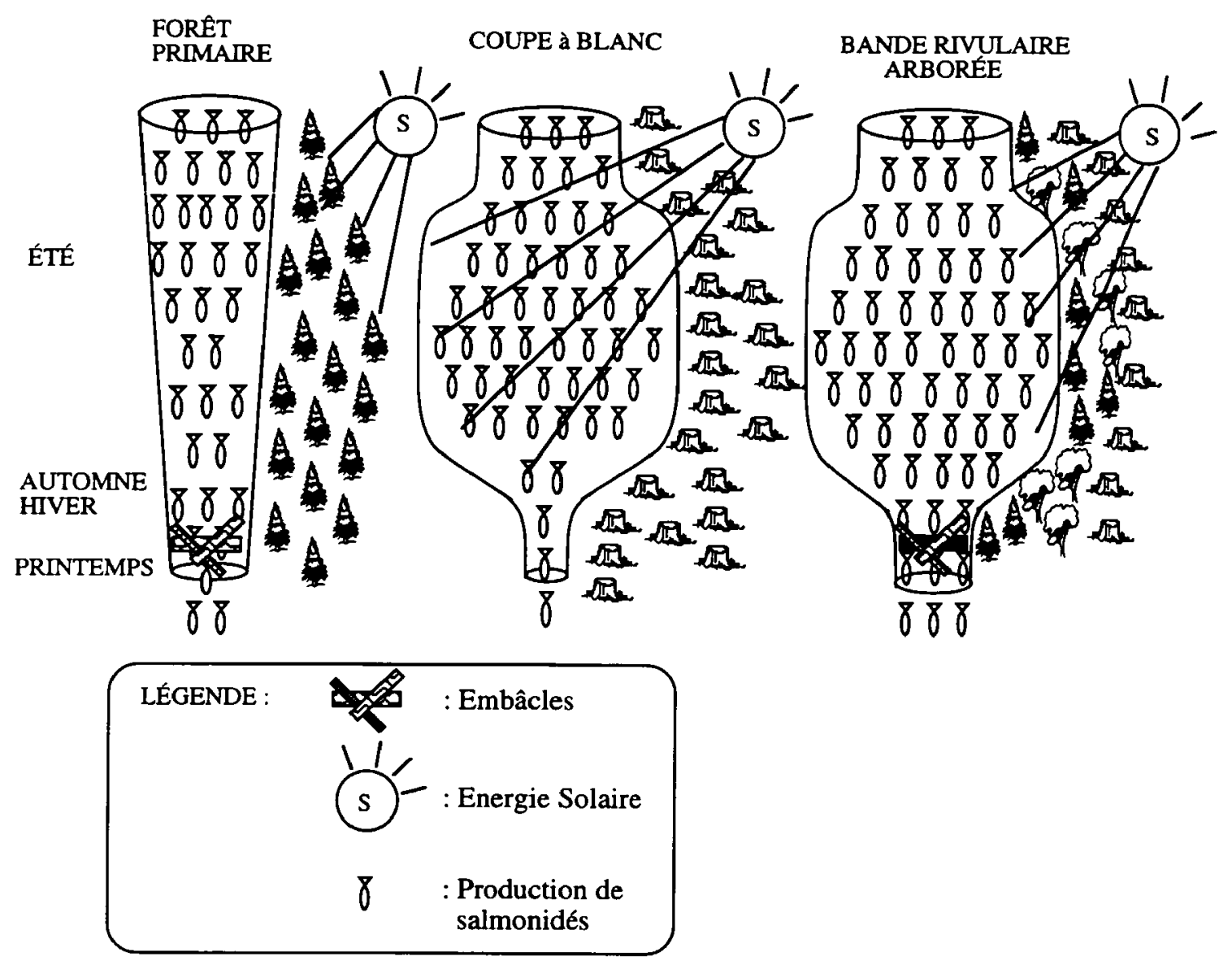

Figure 4 : Influence de la végétation rivulaire sur la production de salmonidés dans des cours d'eau (d'après MURPHY et MEEHAN, 1991, modifié).

Figure 4 : Influence of riparian vegetation on stream production of coho salmon (adapted from MURPHY and MEEHAN, 1991).

- d'établir préalablement une typologie des hydrosystèmes. Systèmes biomorphodynamiques spécifiques, la rivière de plaine océanique, le cours d'eau de piémont à forte énergie ou le torrent montagnard forestier doivent être appréciés spécifiquement par le gestionnaire. II s'agit d'individualiser les hydrosystèmes pour optimiser leurs ressources potentielles.

- de prendre en compte les connectivités et la variabilité spatiale et temporelle de I'hydrosystème dans les programmes futurs de gestion (NAIMAN et al.,1991). II ne faut pas confondre équilibre et stabilité de l'écosystème, la stabilité étant un objectif d'usage. II faut gérer les instabilités localisées, car elles participent à l'équilibre global (AMOROS et al., 1987), et aborder en termes opérationnels les concepts de liberté de divagation latérale et de biodynamique, le bois mort étant alors un élément esthétique, structurel et fonctionnel à retenir dans une gestion réfléchie des hydrosystèmes.

- de définir les problèmes et les objectifs spécifiques à chacune des rivières pour une gestion intégrée de ces milieux (Agence de l'Eau R.M.C., 1991 ; WASSON, 1992).

D'un point de vue pratique, trois règles de gestion peuvent être avancées :

- maintenir une bande rivulaire arborée de qualité. C'est une stratégie reconnue de diversification et donc d'enrichissement piscicoles des écosystèmes d'eau courante (MURPHY et al., 1986 ; THEDINGA et al., 1989 ; NAIMAN et al., 1993). Elle permet la 
filtration des apports latéraux d'origine agricole. La question de la largeur de cette zone reste posée. NEWBOLD (1977) ou PINAY \& DÉCAMPS (1988) suggèrent la conservation d'une bande rivulaire supérieure à $30 \mathrm{~m}$ sur des cours d'eau larges, CULP \& DAVIES (1983) d'une dizaine de mètres, CORBETT \& LYNCH (1985) de 12 à 30 m suivant le bassin versant et les objectifs définis. OSBORNE \& KOVACIC (1991) proposent un éventail de largeurs de 5 à $50 \mathrm{~m}$.

- limiter l'entretien aux formations végétales arborées riveraines les plus anthropisées. Les cours d'eau naturels du Nord-Ouest américain présentent une forte productivité liée à la présence d'embâcles. Le nettoyage des lits et des berges (arbres susceptibles de tomber et de former des embâcles) induit des effets biologiques négatifs : réduction des habitats et des stocks nutritifs non compensés par une augmentation de la production autotrophe obtenue lors de l'ouverture de la canopée.

- préconiser une politique de non-intervention ou de renaturation sur certains tronçons. Elle permettra de retrouver un fonctionnement dans lequel les interconnexions lit mineur / formations végétales arborées riveraines seraient optimales. Elle favorisera la diversification des habitats. Le concept de reproduction naturelle du poisson tend d'ailleurs à se diffuser dans les associations halieutiques (LAFAGE, 1992).

\section{REMERCIEMENTS}

Les auteurs tiennent à remercier J.G. WASSON, Y. SOUCHON et M. PHILIPPE (CEMAGREF de Lyon), P. DUPONT et C. PETIT (Agence de l'Eau Rhône-MéditerranéeCorse), J.P. BRAVARD (Professeur à l'Université Lyon III), C. AMOROS et J. MATHIEU (Professeurs à l'Université Lyon I), J. HAURY (INRA de Rennes), et B. LACHAT (Bureau d'études BIOTEC), qui ont soutenu leur démarche, relu et critiqué le manuscrit. Ils remercient également C. LASNIER (Dessinateur-cartographe à l'Agence de l'Eau RhôneMéditerranée-Corse) pour l'esthétisme des figures.

\section{BIBLIOGRAPHIE}

AGENCE DE L'EAU RHÔNE-MÉdITERRANÉE-CORSE, 1991. Eaux. Lyon, 331 p.

AMOROS C., RICHARDOT - COULET M., PAUTOU G., 1982. Les ensembles fonctionnels : des entités écologiques qui traduisent l'évolution de l'hydrosystème en intégrant le géomorphologique et l'anthropisation (exemple du Haut-Rhône français). Revue de Géographie de Lyon, 57, 49-62.

AMOROS C., ROSTAN J.C., PAUTOU G., BRAVARD J.P., 1987. The reversible process concept applied to the environmental management of large river systems. Env. Manag., 11 (5), 607-617.

AMOROS C., ROUX A.L., 1988. Interaction between water bodies within the floodplains of large rivers : function and development of connectivity. Aus : K.F. Schreiber (Hrsg): Connectivity in Landscape Ecology Proceedings of the 2nd International Seminar of the International Association for Landscape Ecology, Münstersche Geographische Arbeiten, 29, 125-130.

ANDERSON N.H., SEDELL J.R., ROBERTS L.M., TRISKA F.J., 1978. The role of aquatic invertebrates in processing of wood debris in coniferous forest streams. Am. Midl. Nat., 100 (1), 64-82.

ANGERMEIER P.L., KARR J.R., 1984. Relationships between woody debris and fish habitat in a small warmwater stream. Trans. Am. Fish. Soc., 113, 716-726.

BEHMER D.J., HAWKINS C.P., 1986. Effects of overhead canopy on macroinvertebrate production in a Utah stream. Freshwat. Biol., 16, 287-300.

BENKE A.C., HENRY R.L., GILLESPIE D.M., HUNTER R.J., 1985. Importance of snag habitat for animal production in southeastern streams. Fisheries, 10 (5), 8-13. 
BERGERON N., ROY A.G., 1985. Le rôle de la végétation sur la morphologie d'un petit cours d'eau. Géographie Physique et Quaternaire, 39 (1), 323-326.

BESCHTA R.L., BILBY R.E., BROWN G.W., HOLTBY L.B., HOFSTRA T.D., 1987. Stream temperature and aquatic habitat: Fisheries and forestry interactions. In SALKO E.O. et CUNDY T.W. (eds), Forestry and fishery interactions, University of Washington, Institute of forest resources, Seattle, Chapitre 6.

BILBY R.E., 1979. The function and distribution of organic debris dams in forest streams ecosystems. Ph. D. Thesis, Cornell University, Ithaca, New York, 148 p.

BILBY R.E, LIKENS G.E., 1980. Importance of Organic Debris Dams in the Structure and Function of Stream Ecosystem. Ecology, 61, 1107-1113.

BILBY R.E., 1981. Role of organic debris dams in regulating the export of dissolved and particulate matter from a forested watershed. Ecology, 62 (5),1234-1243.

BILBY R.E., WARD J.W., 1989. Changes in characteristics and function of woody debris with increasing size of streams in Western Washington. Trans. Am. Fish. Soc., 118, 368-378.

BILBY R.E., BISSON P.A., 1992. Allochtonous versus autochtonous organic matter contributions to the trophic support of fish populations in clear-cut and old growth forested streams. Can. J. Fish. Aquat. Sci., 49, 540-551.

BISSON P.A., NIELSEN J.L., PALMASON R.A., GROVE L.E., 1981. A system of naming habitat types in small streams, with examples of habitat utilization by salmonids during low streamflow. In ARMANTROUT N.B. (ed.), Acquisition and utilization of aquatic habitat inventory information, Portland, Oregon, American fisheries society, 62-73.

BISSON P.A., BILBY R.E., BRYANT M.D., DOLLOFF C.A., GRETTE G.B., HOUSE R.A., MURPHY M.L., KOSKI K.V., SEDELL J.R., 1987. Large woody debris in forested streams in the Pacific Northwest : past, present and future. In CUNDY T.W. (ed.), Streamside Management : Forestry and Fishery Interactions, Seattle, College of Forest Resources, University of Washington, 143-190.

BOUSSU M.F., 1954. Relationship between trout populations and cover on a small stream. J. Wild. Manag., 18 (2), 229-239.

BRUSVEN M.A., MEEHAN W.R., WARD J., 1986. Summer use of simulated undercut banks by juvenile chinook salmon in an artificial Idaho channel. North Am. J. of Fish. Man., 6, 32-37.

BRYANT M.D., 1981. Organic debris in salmonid habitat in Southeast Alaska: measurement and effects. In ARMANTROUT N.B. (ed.), Acquisition and utilization of aquatic habitat inventory information, Portland, Oregon, American fisheries society, 259-265.

BRYANT M.D., 1983. The role and management of woody debris in west coast salmonid nursery streams. North Am. Journal of Fish. Man., 3, 322-330.

BRYANT M.D., 1985. Changes 30 years after logging in large woody debris, and its use by salmonids. In Riparian Ecosystems and their Management, Fifth North American Riparian Conference, Tucson, Arizona, 329-334 .

BUER K., FORWALTER D., KISSEL M., STHOLER B., 1989. The middle Sacramento river : human impacts on physical and ecological processes along a meandering river. In Proceedings of the California Riparian Systems Conference : Protection, Management and Restoration for the 1990's ; 1988, september 22-24, Davis, U.S.D.A. Forest Service, Gen. Tech. Rep. PSW-110, 22-32 .

BUGERT R.M., BJORNN T.C., MEEHAN W.R., 1991. Summer habitat use by young Salmonids and their responses to cover and predators in a small Southeast Alaska stream. Trans. Am. Fish. Soc., 120, 474 - 485.

BUSTARD D.R., NARVER D.W., 1975. Aspects of the winter ecology of juvenile Coho Salmon and Steelhead Trout. J. Fish. Res. B. Can., 32, 667-680. 
CAMPBELL I.C., DOEG T.J., 1989. Impact of timber harvesting and production on streams : a review. Aust. J. Mar. Freshwater Res., 40, 519-539.

CARBIENER R., 1970. Un exemple de type forestier exceptionnel pour l'Europe occidentale : la forêt du lit majeur du Rhin au niveau du fossé rhénan. Vegetatio, 20, 97-148.

CEMAGREF Bordeaux (Ministère de l'environnement et Ingénierie des Eaux Continentales de Bordeaux), 1988. Gestion des bordures de cours d'eau : Evolutions, Fonctions et Intérêts des ripisylves, en coll. CERR Toulouse et IDF Toulouse, $90 \mathrm{p}$.

CHERGUI H., 1988. Flux et processus de dégradation des apports allochtones particulaires dans un système fluvial : importance relative de l'écotone rives. Thèse de Doctorat, Université Claude Bernard - Lyon I: 146p.

CLEMENT A.M., 1985. Impact des travaux de canalisation sur la faune benthique des cours d'eau en milieu agricole. Ministère Loisir, Chasse et Pêche, Gouvernement du Québec, $64 \mathrm{p}$.

CLIFTON C., 1989. Effects of vegetation and land use on channel morphology. In GRESSWELL R.E. (ed.), Practical approaches to riparian resource management, an educational workshop, Billings, Montana (U.S.A), 121-129.

COPP G.E., OLIVIER J.M., PENAZ M., ROUX A.L., 1992. Juvenile fishes as functional describers of fluvial ecosystem dynamics : applications on the River Rhône, France. Reg. Rivers Res. Manag., 6, 135-145.

CORBETT E.S., LYNCH J.A., 1985. Management of streamside zones on municipal watersheds. In Riparian Ecosystems and their Management, Fifth North American Riparian Conference, Tucson, Arizona, 187-190 .

CRISP D.T., 1989. Some impacts of human activities on trout salmo trutta populations. Freshwat. Biol., 21, 21-33.

CULP J.M., DAVIES R.W., 1983. An assessment of the effects of streambank clear-cutting on macroinvertebrate communities in a managed watershed. Can. Tech. Rep. Fish. Aquati. Sci., 1208, $116 \mathrm{p}$.

CUMMINS K.W., SEDELL J.R., SWANSON F.J., MINSHALL G.W., FISHER S.G., CUSHING C.E., PETERSEN R.C., VANNOTE R.L., 1983. Organic matter budgets for stream ecosystems : problems in their evaluation. In BARNES J.R., MINSHALL G.W. (ed.), Stream Ecology, New York and London 299-353.

DAHLSKOG S., 1966. Sedimentation and vegetation in a Lapland mountain delta. Geographiska Annaler , 48 A, 86-101.

DAWSON F.H., KERN-HANSEN V., 1979. The effect of natural and artificial shade on the macrophytes of lowland streams and the use of shade as a management technique. Int. Revue Ges. Hydrobiol., 64 (4), 437-455.

DAWSON F.H., HASLAM S.M., 1983. The management of river vegetation with particular reference to shading effects of marginal vegetation. Landscape Planning, Biotechnical engineering series, 10, 147-169.

DÉCAMPS H., NAIMAN R.J., 1989. L'écologie des fleuves. La Recherche, 208 (20), 310-319.

DETHIOUX M., 1988. Végétation et protection des berges. Gembloux, Belgique, Centre de recherche et de promotion forestières, $14 \mathrm{p}$.

DOLLOFF C.A., 1986. Effects of cleaning on juvenile Coho Salmon and Dolly Varden in Southeast Alaska. Trans. Am. Fish. Soc., 115, 743-755.

ELLIOTT S.T., 1986. Reduction of a Dolly varden population and macrobenthos after removal of logging debris. Trans. Am. Fish. Soc., 115, 392-400.

EVEREST F.H., REEVES G.H., SEDELL J.R., HOHLER D.B., CAIN T.C., 1988. Changes in habitat and populations of steelhead trout, coho salmon, and chinook salmon in Fish Creek, Oregon, as related to habitat improvement., USDA Forest Service, Annual Report 1987, $115 \mathrm{p}$. 
FAUSCH K.D., NORTHCOTE T.G., 1992. Large woody debris and Salmonid habitat in a small coastal British Columbia stream. Can. J. Fish. Aquat. Sci., 49, 682-693.

FORWARD C.D., 1984. Organic debris complexity and its effect on small scale distribution and abundance of coho fry populations in Carnation Creek, British Columbia. B.S.F. Thesis, Univ. of British Columbia, Vancouver (Canada).

FRANKLIN J.F., 1988. Structural and functional diversity in temperate forests. In WILSON E.O. (ed.), Biodiversity, National Academy Press, Washington, DC, 166-174.

FULLER R.L., FRY T.J., 1991. The influence of temperature and food quality on the growth of Hydropsyche betteni (Trichoptera) and Simulium vittatum (Diptera). J. of Freshwat. Ecol., 6 (1), 75-86.

FUSTEC E., 1988. Le problème des nitrates dans les plaines alluviales, Impact des ripisylves sur l'évolution des teneurs en nitrates dans la nappe et dans le fleuve ; Exemple de la moyenne vallée de la Garonne. Ministère de l'Equipement, du Logement, de l'Aménagement du Territoire et des Transports, $52 \mathrm{p}$.

GARD R., 1961. Creation of trout habitat by constructing small dams. J. Wild. Manag., 52 (4), 384-390.

G.I.R.E.A., 1987. Aménagement écologique des berges des cours d'eau navigables, la berge interface terre-eau : ses caractéristiques, fonctions et utilisations. Belgique, rapport $n^{\circ} 1$. Ministère des travaux publics, $68 \mathrm{p}$.

GORMAN T.O., KARR J.R., 1978. Habitat structure and stream fish communities. Ecology, 59 (3), 507-515.

GREGORY J.D., STOKOE J.L., 1981. Streambank Management. In Warmwater streams symposium, American Fisheries Society, 276-281.

GREGORY K.J., GURNELL A.M., HILL C.T., 1985. The permanence of debris dams related to river channel processes. Hydrological Sciences Journal, 30 (3), 371-381.

GREGORY K.J., GURNELL A.M., 1988. Vegetation and river channel form and process. In VILES H. (ed.), Biogeomorphology, Oxford, 11-42.

GREGORY S.V., LAMBERTI G.A., MOORE K. M.S., 1989. Influence of valley floor landforms on stream ecosystems. In Proceedings of the California Riparian Systems Conference, Protection, Management and Restoration for the 1990's ; 1988, september 22 -24, Davis, U.S.D.A Forest Service, Gen. Tech. Rep. PSW110, 3-8 .

GREGORY K.J., 1992. Vegetation and river channel process interactions. In BOON P.J., CALOW P., PETTS G.E. (ed.), River Conservation and Management , 255-269.

GREGORY K.J., DAVIS R.J., 1992. Coarse woody debris in stream channels in relation to river channel management in woodland areas. Reg. Rivers Res. Manag., 7, 117-136.

GRETTE G.B., 1982. The role of large organic debris in juvenile salmonid rearing habitat in small streams. M.S. Thesis, Univ. of Washington, Seattle, $105 \mathrm{p}$.

GURNELL A.M., GREGORY K.J., 1984. The influence of vegetation on stream channel processes. In BURNT T.P., WALLING D.E. (ed.), Catchment experiments in geomorphology, 515-535.

HADLEY R.F., 1961. Influence of riparian vegetation on channel shape, northeastern Arizona. US Geol. Surv. Prof. Paper, 424 C, 30-31.

HALL J.D., LANTZ R.L., 1969. Effects of logging on the habitat of coho salmon and cutthroat trout in coastal streams. In Symposium on salmon and trout in streams, University of British Columbia, Vancouver, 355-375.

HARMON M.E., FRANKLIN J.F., SWANSON F.J., SOLLINS P., GREGORY S.V., LATTIN J.D., ANDERSON N.H., CLINE S.P., AUMEN N.G., SEDELL J.R., LIENKAEMPER G.W., CROMACK Jr. K., CUMMINS K.W., 1986. Ecology of coarse woody debris in temperate ecosystems. In MacFadayen A., Ford E.D. (ed.), Advances in ecological research., Academic Press, London, 133-302. 
HARR R.D., LEVNO A., MERSEREAU R., 1982. Streamflow changes after logging 130 year old douglas fir in two small watersheds. Water Res. Res., 18 (3), 637-644.

HAURY J., 1985. Etude écologique des macrophytes du Scorff (Bretagne Sud). Thèse Docteur Ingénieur, U.E.R. Sciences de la vie et de l'environnement, Rennes I, 239 p.

HAWKINS C.P., MURPHY M.L., ANDERSON N.H., 1982. Effects of canopy, substrate composition, and gradient on the structure of macroinvertebrate communities in cascade range streams of Oregon. Ecology, 63 (6), 1840-1856.

HAWKINS C.P., MURPHY M.L., ANDERSON N.H., WILZBACH M.A., 1983. Density of fish and salamanders in relation to riparian canopy and physical habitat in streams of the Northwestern United States. Can. J. Fish. Aquat. Sci., 40, 1173-1185.

HEEDE B.H., 1972. Influence of a forest on the hydraulic geometry of two mountain streams. Water Res.Bull., 8, 523-530.

HEEDE B.H., 1985. Interactions between streamside vegetation and stream dynamics. U.S.D.A. Forest Service, Gen. Tech. Rep. RM120, 54-58.

HEIFETZ J., MURPHY M.L., KOSKI K.V., 1986. Effects of logging on winter habitat of juvenile salmonids in alaskan streams. North Am. J. of Fish. Man., 6, 52-58.

HICKIN E.J., 1984. Vegetation and river channel dynamics. Canadian Geographer, 28 (2), 110-126.

HICKIN E.J., NANSON G.C., 1984. Lateral migrations of river bends. Journal of Hydraulic Engineering, 110, 11p.

HICKS B.J., HALL J.D., BISSON P.A., SEDELL J.R., 1991. Responses of salmonids to habitat changes. In MEEHAN W.R. (ed.), Influence of forest and rangeland management on salmonid fishes and their habitats, 483-518.

HOLMAN G., EVANS W.A., 1964. Stream clearance project completion report for Noyo River, Mendocino county. In Sacramento, California Department of Fish and Game, report 64-10.

HOLOPAINEN A.L., HUTTUNEN P., AHTIAINEN M., 1991. Effects of forestry practices on water quality and primary productivity in small forest brooks. Verh. Internat. Verein. Limnol., 24, 1760-1766.

HOUSE R.A., BOEHNE P.L., 1986. Effects of instream structures on salmonid habitat and populations in Tobe Creek, Oregon. North Am. J. of Fish. Man., 6, 38-46.

HUNTER C.J., 1991. Better trout habitat, a guide to stream restoration and management, PALMER T. (ed.), Montana Land Reliance, $320 \mathrm{p}$.

HUPP C.R., Osterkamp W.R., 1985. Bottomland vegetation distribution along Passage Creek, Virginia, in relation to fluvial landforms. Ecology, 63 (6), 670-681.

JOHNSON S.W., HEIFETZ J., 1985. Methods for assessing effects of timber harvest on small streams. National Marine Fisheries Service, $33 p$.

JOHNSTON C.A., NAIMAN R.J., 1987. Boundary dynamics at the aquatic-terrestrial interface, the influence of beaver and geomorphology. Landscape Ecology, 1, 47-57.

KELLER E.A., SWANSON F.J., 1979. Effects of large organic material on channel form and fluvial processes. Earth Surf. Proc. Land., 4, 361-380.

KELLER E.A., TALLY T., 1979. Effects of large organic debris on channel form and fluvial processes in the coastal redwood environment. In Adjustments of the fluvial syst. Proceedings tenth annual geomorphology, Symposium serie, New York, 169-197.

KELLER E.A., MAC DONALD A., 1983. Large organic debris and anadromous fish habitat in the coastal redwood environment: the hydrologic system, Univ. of California, Davis, Technical Completion Report, Office of the Director California Water Resources Center, $48 \mathrm{p}$. 
KOUWEN N., RUH-MING L., 1980. Biomechanics of vegetative channel linings. Journal of the hydraulics division, 106 (HY6), 1085-1103.

LACHAT B., 1991. Le cours d'eau, Conservation, Entretien et Aménagement. Conseil de l'Europe, Comité directeur pour la protection et la gestion de l'environnement et du milieu naturel, Strasbourg, $84 \mathrm{p}$.

LAFAGE J.P., 1992. La pêche en France, un potentiel à libérer. Eaux libres, Conseil Supérieur de la Pêche, 10, 19-23.

LIENKAEMPER G.W., SWANSON F.J., 1986. Dynamics of large woody debris in streams in old growth douglas-fir forests. Can. J. For. Res., 17, 150-156.

LISLE T.E., 1982. Roughness elements : a key resource to improve anadromous fish habitat. In HASSLER T.J. (ed.), Proceedings propagation, enhancement and rehabilitation of anadromous salmonid populations and habitat in the Pacific Northwest Symposium, 93-98.

LYELL C., 1969. Principles of Geology, New York, The Sources of Science $n^{\circ}$ 84, Vol. 2.

LYNCH J.A., CORBETT E.S., HOOPES R., 1977. Implications of forest management practices on the aquatic environment. Fisheries, 2 (2), 16-22.

MARSTON R.A., 1982. The geomorphic significance of log steps in forest streams. Annals of Association of American Geographers, 72 (1), 99-108.

MASER C., TARRANT R.F., TRAPPE J.M., FRANKLIN J.F., 1988. From the forest to the sea, a story of fallen trees, Pacific Northwest Research Station, U.S.D.A. Forest Service, General Technical Report PNW GTR 229, Portland, Oregon,120p.

MEEHAN W.R., SWANSON F.J., SEDELL J.R., 1977. Influence of riparian vegetation on aquatic ecosystems with particular references to salmonid fishes and their food supply. In USDA Forest Service, Gen. Tech. Rep. RM 43, Importance, preservation and management of riparian habitat, 137-145.

MEEHAN W.R., BRUSVEN M.E., WARD J.F., 1987. Effects of artificial shading on distribution and abundance of juvenile chinook salmon. Great Basin Naturalist, 47 (1), 22-31.

MERRELL T.R., 1951. Stream improvement as conducted in Oregon on the Clatskanie River and tributaries. Fish Comm. Oregon Res. Briefs, 3, 41-47.

MOORE M.S., GREGORY S.V., 1989. Geomorphic and riparian influences on the distribution and abundance of salmonids in a cascade mountain stream. In Proceedings of the California Riparian Systems Conference, Protection, Management and Restoration for the 1990's ; 1988, september 22-24, Gen. Tech. Rep. PSW 110, Davis, U.S.D.A. Forest Service, 256-261

MORING J.R., GARMAN G.C., MULLEN D.M., 1985. The value of riparian zones for protecting aquatic systems : general concerns and recent studies in Maine. In Riparian Ecosystems and their Management, Fifth North American Riparian Conference, Tucson, Arizona, 315-319.

MORTENSEN E., 1977. Density dependent mortality of trout fry and its relationship to the management of small streams. J. Fish. Biol., 11, 613-617.

MOSLEY P., 1981. The influence of organic debris on channel morphology and bedload transport in a New Zealand forest stream. Earth Surf. Proc. Land., 6, 571-579.

MURPHY M.L., HAWKINS C.P., ANDERSON N.H., 1981. Effects of canopy modification and accumulated sediment on stream communities. Trans. Am. Fish. Soc., 110, 469-478.

MURPHY M.L., KOSKY K.V., HEIFETZ J., JOHNSON S.W., KIRCHHOFER D., THEDINGA J.F., 1984. Role of large organic debris as winter habitat of juvenile salmonids in alaskan streams. In Proceedings Western Association of Fish and Wildlife Agencies, 251-262. 
MURPHY M.L., HEIFETZ J., JOHNSON S.W., KOSKI K.V., THEDINGA J.F., 1986. Effects of clearcut logging with and without buffer strips on juvenile salmonids in Alaskan streams. Can. J. Fish. Aquat. Sci., 43, 1521-1533.

MURPHY M.L., MEEHAN W.R., 1991. Stream ecosystems. In MEEHAN W.R. (ed.), Influences of forest and rangeland management on salmonid fishes and their habitats, Bethesda, Maryland, USA, 17-46.

NAIMAN R.J., JOHNSTON C.A., KELLEY J.C., 1988. Alteration of North American streams by beaver. The structure and dynamics of streams are changing as beavers recolonize their historic habitat. Bioscience, 38 (11), 753-762.

NAIMAN R.J., DÉCAMPS H., FOURNIER F., 1989. Role of land/inland water ecotones in landscape management and restoration ; proposals for collaborative research, Paris, MAB Digest 4, $93 \mathrm{p}$.

NAIMAN R.J., DÉCAMPS H., 1990. The ecology and management of aquatic-terrestrial ecotones, Paris, Man and the Biosphere series, Unesco, 4, $316 \mathrm{p}$.

NAIMAN R.J., STANFORD J.A., DÉCAMPS H., 1991. The application of ecological knowledge to river management. Communication au colloque "Quels fleuves pour demain» Orléans. $47 \mathrm{p}$.

NAIMAN R.J., DÉCAMPS H., POLLOCK M., 1993. The role of riparian corridors in maintaining regional biodiversity. Ecological Applications, 3 (2), 209-212.

NANSON G.C., 1977. Channel migration, floodplain formation and vegetation succession on a meandering river floodplain in N.E. British Columbia, Canada. Ph. D. Thesis, Simon Fraser Univ., Burnaby, British Columbia, 349 p.

NANSON G.C., BEACH H.F., 1977. Forest succession and sedimentation on a meandering river floodplain, Northeast British Columbia, Canada. Journal of Biogeography, 4, 229-251.

NEWBOLD J.D., 1977. The use of macroinvertebrates as indicators of logging impacts on streams, with an evaluation of buffer strip effectiveness. Ph. D. Thesis, University of California, Berkeley, CA, $103 p$.

O'CONNOR M.D., ZIEMER R.R., 1989. Coarse woody debris ecology in a second growth sequoia sempervirens forest stream. In Proceedings of the California Riparian Systems Conference, Protection, Management and Restoration for the 1990's ; 1988, september 22-24, Davis, U.S.D.A. Forest Service, Gen. Tech. Rep. PSW110, 165-171.

O'CONNOR N.A., 1992. Quantification of submerged wood in a lowland Australian stream system. Freshwat. Biol., 27, 387-395.

OSBORNE L.L., KOVACIC D.A., 1991. Riparian vegetated buffer strips in stream restoration and management. In International Workshop on Lowland Stream Restoration, Lund, $19 \mathrm{p}$.

PAUTOU G., DÉCAMPS H., 1985. Ecological interactions between the alluvial forests and hydrology of the Upper Rhône. Arch. Hydrobiol., 104 (1), 13-37.

PETERJOHN W.T., CORREL D.L., 1984. Nutrient dynamics in an agricultural watershed, observations on the role of a riparian forest. Ecology, 65 (5), 1466-1475.

PETERSEN R.C., PETERSEN L.B., LACOURSIÈRE J., 1992. A building block model for stream restoration. In BOON P.J., CALOW P., PETTS G.E. (ed.), River Conservation and Management, $13 \mathrm{p}$.

PETTS G.E., 1990. The role of ecotones in aquatic landscape management. In NAIMAN J., DÉCAMPS $H$. (ed.), The ecology and management of aquatic-terrestrial ecotones, Unesco, 227-261.

PIÉGAY H., 1993. The nature, mass and preferential sites of coarse woody debris deposits in the lower Ain valley (Mollon reach), France. Reg. Rivers Reg. Manag., 8, 359-372. 
PIÉGAY H., BRAVARD J.P., 1993. Processus biomorphodynamiques et métamorphose fluviale : exemple du secteur de Mollon dans la plaine alluviale de la basse vallée de l'Ain. Rev. Géomorph. Dyn., 4, 123-138.

PINAY G., 1986. Impact of a riparian forest on the nitrogen content of phreatic water in the Garonne basin. In LAUGA J., DÉCAMPS H., HOLLAND M.M. (ed.), Land use impacts on aquatic ecosystems, MAB UNESCO, PIREN-CNRS, 303-317.

PINAY G., DÉCAMPS H., 1988. The role of riparian woods in regulating nitrogen fluxes between the alluvial aquifer and surface water. A conceptual model. Reg. Rivers Res. Manag., 2, 507-516.

PLATTS W.S., NELSON R.L., 1989. Characteristics of riparian plant communities and streambanks with respect to grazing in Northeastern Utah. In GRESSWELL R.E. (ed.), Practical approaches to riparian resource management, an educational workshop, Billings, Montana (U.S.A.), 73-81.

PLATTS W.S., 1991. Livestock grazing. In MEEHAN W.R. (ed.), Influences of forest and rangeland management on salmonid fishes and their habitats, 389-423.

POUJARDIEU A., 1988. Gestion de la ripisylve des cours d'eau. Mém. ENITA, CEMAGREF-D.Q.E.P.P., Bordeaux, 2 tomes, $94 \mathrm{p}+$ annexes.

PROCHAZKA K., STEWART B.A., 1991. Leaf litter retention and its implications for shredder distribution in two headwater streams. Arch. Hydrobiol., 120 (3), 315-325.

QUINN J.M., WILLIAMSON R.B., SMITH R.K., VICKERS M.L., 1992. Effects of riparian grazing and channelisation on streams in Southland, New Zealand. 2. Benthic invertebrates. New Zealand Journal of Marine and Freshwater Research , 26, 259273.

RHODES J., SKAU C.M., GREENLEE D., BROWN D.L., 1985. Quantification of nitrate uptake by riparian forests and wetlands in an undisturbed headwaters watershed. In Riparian Ecosystems and their Management, Fifth North American Riparian Conference, Tucson, Arizona, 175-179.

ROBISON G.E., 1987. Large woody debris and channel morphology of undisturbed streams in Southeast Alaska. Master of Science in Forest Engineering, Oregon State University, $125 \mathrm{p}$.

ROBISON G.E., BESCHTA R.L., 1990. Coarse woody debris and channel morphology interactions for undisturbed streams in Southeast Alaska, USA. Earth Surf. Proc. Land., 15, 149-156.

SCHUMM S.A., 1977. The fluvial system, NewYork, J. Wiley and Sons Ltd, 338 p.

SEDELL J.R., LUCHESSA K.J., 1982. Using the historical record as an aid to salmonid habitat enhancement. In Proc. of a Symp. on Acquisition and Utilization of Aquatic Habitat Inventory Information Held, Portland, Oregon (U.S.A.), 210-223 .

SEDELL J.R., FROGGATT J.L., 1984. Importance of streamside forests to large rivers, the isolation of the Willamette River (Oregon, USA) from its floodplain by snagging and streamside forest removal. Verhandlungen Inter. Vereinigung Limnologie, 22, 18281834.

SEDELL J.R., YUSKA J.E., SPEAKER R.W., 1984. Habitats and salmonid distribution in pristine, sediment rich river valley systems : South Fork Hoh and Queets River, Olympic National Park. In Fish and Wildlife relationships in oldgrowth forests, American Institute of Fishery Research Biologists, 33-46.

SEDELL J.R., SWANSON F.J., GREGORY S.V., 1985. Evaluating fish response to woody debris. In Pacific northwest stream habitat management workshop, Arcata, California, Humboldt State University, 222-245.

SEDELL J.R., EVEREST F.H., GIBBONS D.R., 1987. Streamside vegetation management for aquatic habitat. In Proc. of the National Silviculture Workshop, Sacramento, California, 115125 . 
SEDELL J.R., BISSON P.A., SWANSON F.J., GREGORY S.V., 1988. What we know about large trees that fall into streams and rivers? In MASER C., TARRANT R.F., TRAPPE J.M., FRANKLIN J.F. (ed.), From the forest to the sea, a story of fallen trees, Pacific Northwest Research Station, U.S.D.A. Forest Service, General Technical Report PNWGTR 229, Portland, Oregon (U.S.A.), Chap. 3, 47-81

SIOLI H., 1982. Tropische Flusse in ihren Beziehungen zur terrestrischen Umgebung und im Hinblick auf menschliche Eingriffe. Arch. Hydrobiol., 95, 463-485.

SMITH G.D., 1976. Effect of vegetation on lateral migration of anastomosed channels of a glacier Meltwater River. Geological Society of American Bulletin, 87, 857-860.

SMITH C.M., 1987. Sediment, phosphorus, and nitrogen in channellized surface run off from a New Zealand pastoral catchment. New Zealand Journal of Marine and Freshwater Research, 21, 627-639.

SMITH C.M., 1989. Riparian pasture retirement effects on sediment, phosporus and nitrogen in channellized surface run off from pastures. New Zealand Journal of Marine and Freshwater Research, 23, 139-146.

SPOTTS R., 1989. Conflicts in river management : a conservationist's perspective on Sacramento river riparian habitats impacts, threats, remedies, opportunities, and consensus. In Proceedings of the California Riparian Systems Conference, Protection, Management and Restoration for the 1990's ; Gen. Tech. Rep. PSW 110 , Davis, U.S.D.A. Forest Service, 521-525 .

STEINMAN A.D., MCINTIRE C.D., LOWRY R.R., 1988. Effects of irradiance and age chemical constituents of algal assemblages in laboratory streams. Arch. Hydrobiol., $114(1), 45-61$.

STRAHLER A.N., 1957. Quantitative analysis of watershed geomorphology. Trans. Amer. Geophys. Union, 38, 913-920.

SWANSON F.J., LIENKAEMPER G.W., 1978. Physical consequences of large organic debris in Pacific Northwest Streams, U.S.D.A. Forest Service, Portland, Oregon (U.S.A.), 12 p.

SWANSON F.J., LIENKAEMPER G.W., 1982. Interactions among fluvial processes, forest vegetation and aquatic ecosystems, South Fork Hoh River, Olympic National Park. In FRANKLIN J.F., STARKEY E.E., MATTHEWS J.E. (ed.), Ecological Research in National Parks of the Pacific Northwest, Oregon State Univ., 30-34 .

THEDINGA J.F., MURPHY M.L., HEIFETZ J., KOSKI K.V., JOHNSON S.W., 1989. Effects of logging on size and age composition of juvenile coho salmon (Oncorhynchus kisutch) and density of presmolts in Southeast Alaska streams. Can. J. Fish. Aquat. Sci., 46, 1383-1391.

TOEWS D.A.A., MOORE M.K., 1982. The effects of streamside logging on large organic debris in Carnation Creek. Province of British Columbia, Ministry of Forest, $30 \mathrm{p}$.

TREMOLIĖRES M., CARBIENER R., 1982. Aspects de l'impact de litières forestières sur des écosystèmes aquatiques. II. Evolution à moyen et long terme de l'activité de désoxygénation. De quelques propriétés des phytomélanines formées. Acta Oecologica / Oecologia Generalis, 3 (2), 241-257.

TRISKA F.J., CROMACK K., 1980. The role of wood debris in forests and streams. In WARING R.H. (ed.), Forests, Fresh perspectives from ecosystem analysis. Oregon State University Press, Corvallis, Oregon (U.S.A.), 171-190.

TRISKA F.J., SEDELL J.R., GREGORY S.V., 1982. Coniferous forest streams. In Analysis of coniferous forest ecosystems in the Western United States, Pennsylvania, 292-332.

TRISKA F.J., 1984. Role of wood debris in modifying channel geomorphology and riparian areas of a large lowland river under pristine conditions, a historical case study. Verh. Internat. Verein. Limnol., 22, 1876-1892. 
TSCHAPLINSKI P.J., HARTMAN G.F., 1983. Winter distribution of juvenile Coho Salmon before and after logging in Carnation Creek, British Columbia, and some implications for overwintering survival. Can. J. Fish. Aquat. Sci., 40, 452-461.

VANNOTE R.L., MINSHALL G.W., CUMMINS K.W., SEDELL J.R., CUSHING C.E., 1980. The River Continuum Concept. Can. J. of Fish. Aquat Sci., 37, 130-137.

WALLACE J.B., BENKE A.C., 1984. Quantification of wood habitat in subtropical coastal plain streams. Can. J. Fish. Aquat. Sci., 41, 1643-1652.

WALTER J.M., 1992. Pour une forêt naturelle. Bull. Soc. Ind. de Mulhouse, Les espaces naturels rhénans, 824 (1), 277-279.

WARD G.M., CUMMINS K.W., GREGORY S.V., SPEAKER R.W., DUDLEY T.L., WARD A.K., 1982. Habitat and food resources for invertebrate communities in South Fork Hoh River, Olympic National Park, Washington. In Proceedings of the Second Conference on Scientific Research in National Parks, Washington, 35-46.

WASSON J.G., 1992. Les orientations fondamentales par bassin, propositions pour une gestion intégrée des écosystèmes d'eau courante. CEMAGREF Lyon, BEA/LHQ, 32 p.

WILZBACH M.A., 1985. Relative roles of food abundance and cover in determining the habitat distribution of stream Dwelling Cutthroat trout (Salmo clarki). Can. J. Fish. Aquat. Sci., 42, 1668-1672.

WILZBACH M.A., HALL J.D., 1985. Prey availability and foraging behavior of cutthroat trout in an open and forested section of stream. Verh. Internat. Verein. Limnol., 22, 25162522.

WILZBACH M.A., CUMMINS K.W., HALL J.D., 1986. Influence of habitat manipulations on interactions between cutthroat trout and invertebrate drift. Ecology, 64 (4), 898-911.

WITT A., 1985. Vegetational influences on intrachannel deposition : evidence from the Konczak stream, greater poland lowlands, Western Poland. Quaestiones geographicae, 9, 145-160.

ZALEWSKI M., THORPE J.E., GAUDIN P., 1991. Fish and land/inlandwater ecotones, MAB, Unesco, University of Lodz, Stirling and Lyon I, $102 \mathrm{p}$.

ZIMMERMAN R.C., GOODLETT J.C., COMER G.H., 1967. Influence of vegetation on channel form of small streams. In Symposium on river morphology, Wallingford, England, 255-275. 